\title{
2 Die Etablierung einer organisierten Wirtschaftswerbung
}

\section{1 Ökonomische Bedingungen, technologische Innovationen und neue kommunikative Herausforderungen}

Im Verlauf des 19. Jahrhunderts vollzog sich Zug um Zug die Industrialisierung Österreich-Ungarns. Als gesamtökonomisch nachteilig erwiesen sich allerdings ein vergleichsweise niedriges Wirtschaftswachstum der Monarchie sowie eine nicht gleichmäßige Industrialisierung des ganzen Landes. Fortschrittshemmend waren zudem die überbordende Regelungsfreude der Bürokratie sowie der Mangel an Privatinitiative und eine allgemeine Kapitalismusfeindlichkeit nach dem Börsenkrach von 1873. ${ }^{35}$ Ende der 1880er-Jahre war in Österreich-Ungarn allmählich eine Konjunkturbelebung zu verzeichnen. Beginnend mit 1896 setzte ein lang anhaltender wirtschaftlicher Aufschwung ein. Diese „Zweite Gründerzeit“ wurde vor allem von den Eisen- und Stahlunternehmen gestützt, zugleich konnte aber auch die Nahrungs- und Genussmittelbranche in der Residenzstadt hohe Zuwachsraten verzeichnen. Neue Leitsektoren, wie etwa die Elektro-, die Fahrzeug- und die Großchemieindustrie, expandierten. ${ }^{36}$

Parallel dazu erfolgte eine zunehmende Konzentrationsbewegung in einzelnen Branchen. So kam es 1881 etwa zur Zusammenführung der steirischen und der Kärntner Eisen- und Stahlindustrie in die Österreichisch-Alpine Montangesellschaft. Ähnliche Bestrebungen gab es im Bereich der Bier-, Branntwein- und Zuckererzeugung sowie der aufstrebenden Chemie-, Fahrzeug-, Reifen- und Elektrobranche. ${ }^{37}$ Auf diese Weise konnten große Kapitalmengen akkumuliert werden, die für den Ausbau moderner Produktionseinheiten aufzubringen waren, sowie Kosten im Bereich der Organisation und Fabrikation reduziert werden. Große, oft marktdominierende Unternehmen schlossen sich zu Kartellen zusammen, trafen Produktions- und Marktabsprachen, um ihre Vormachtstellung zu sichern und auszubauen. Getragen wurde diese wachsende Machtkonzentration von den führenden Bankinstituten, die sich gegen Ende des 19. Jahrhunderts intensiv an industriellen Unternehmungen beteiligten. Staatliche Eingriffe über Schutzzölle oder Subventionen verdichteten das sich etablierende

35 Leidinger, Hannes/Moritz, Verena/Moser, Karin: 1896-1918. Das Ende der Habsburgermonarchie, Booklet zur DVD-Edition, Teil 1: Moser, Karin/Leidinger, Hannes/Moritz, Verena/Achenbach, Michael: Die Österreich-Box. Ein Jahrhundert Zeitgeschichte in originalen Filmdokumenten 1896-1995, Wien 2010, S. 19 f.

36 Sandgruber, Roman: Ökonomie und Politik. Österreichische Wirtschaftsgeschichte vom Mittelalter bis zur Gegenwart, Wien 1995, S. 292.

37 Ebd., S. 294. 
Machtgeflecht zwischen Bürokratie, Industrie und partizipierenden Geldinstituten. Eine „Organisierung des Kapitalismus“ war erfolgt. ${ }^{38}$

Die neuen Energieträger Petroleum, Gas und Elektrizität veränderten den Alltag und die Arbeitswelt. ${ }^{39}$ Durch das künstliche Licht wurde der Tag länger, die Produktions- und Arbeitsabläufe in den Fabriken änderten sich tiefgreifend. Moderne Motorenantriebe kamen zum Einsatz, kleinere automatische Arbeitseinheiten entstanden und leiteten das Fließbandzeitalter ein. Die standardisierte Herstellung von Produkten war möglich und führte im ausgehenden 19. Jahrhundert zur Einführung erster einheitlicher Normierungen bei komplexen Stückgütern (etwa bei Fahrrädern, Nähmaschinen, Waffen etc.). ${ }^{40}$ Diese Entwicklung wies bereits auf eine zunehmende Globalisierung des Warenverkehrs hin, die auch von der weiter wachsenden Mobilisierung getragen wurde. Moderne Elektromotoren erweiterten die Transportmöglichkeiten und die Erreichbarkeit potenzieller Märkte.

Diese neuen Bedingungen forderten von den Unternehmern eine neue strategische Ausrichtung, die sich sukzessive weniger an Technologien und Produkten und mehr an Absatzmaßnahmen orientierte. Erste entsprechende Schritte wurden bereits im letzten Drittel des 19. Jahrhunderts gesetzt. Das Anzeigenwesen erlebte einen Aufschwung, Plakate dominierten immer mehr das Straßenbild, die Schaufenster und Verkaufsräume wurden arrangiert und inszeniert. ${ }^{41}$ Als Reaktion auf das vielfältigere Warenangebot und zur klaren Abgrenzung von Produkten aus dem gleichen Sortiment wurde bereits vor 1900 der Markenartikel eingeführt. Alleinstellungsmerkmale wie Qualität, standardisierte Produktbeschaffenheit, Mengengröße, Verpackung und Warensignet wurden festgelegt und aufwendig beworben. ${ }^{42}$ Parallel dazu richteten einzelne

38 Ebd., S. 293-297. Eigner, Peter/Helige, Andrea (Hg.): Österreichische Wirtschafts- und Sozialgeschichte im 19. und 20. Jahrhundert, Wien/München 1999, S. 91 f. Leidinger, Kapitalismus, Wien/ Köln/Weimar 2008, S. 70 f.

39 Die Autorin zitiert hier Kapitel 1 ihres Artikels: Moser, Karin: Filmischer Aufbruch in die Moderne: Technik, Mobilität und Masse, Kapitel 1 „Filmische Faszination - die Maschine in der Kriegspropaganda“, in: Virtuelle Ausstellung „Der Erste Weltkrieg und das Ende der Habsburgermonarchie, http://ww1.habsburger.net/de/kapitel/filmische-faszination-die-maschine-der-kriegspropaganda, 30.11.2014.

40 Eigner/Helige: Wirtschafts- und Sozialgeschichte, S. 98. Weitensfelder, Hubert: Technischer Wandel und Konsum im 19. und frühen 20. Jahrhundert, in: Breuss, Susanne/Eder, Franz X. (Hg.): Konsumieren in Österreich 19. und 20. Jahrhundert, Wien 2006, S. 118 f.

41 Rossfeld, Roman: Markenherrschaft und Reklameschwung. Die schweizerische Schokoladenindustrie zwischen Produktion- und Marketingorientierung, 1860-1914, in: Berghoff, Hartmut (Hg.): Marketinggeschichte. Die Genese einer modernen Sozialtechnik, Frankfurt/New York 2007, S. 88. Eder, Franz X.: Geschichte des Konsumierens. Ansätze und Perspektiven der (historischen) Konsumforschung, in: Breuss, Susanne/Eder, Franz X. (Hg.), Konsumieren in Österreich 19. und 20. Jahrhundert, Wien 2006, S. 26.

42 Eigner, Peter: (Detail)Handel und Konsum in Österreich im 20. Jahrhundert. Die Geschichte einer Wechselbeziehung, in: Breuss, Susanne/Eder, Franz X. (Hg.): Konsumieren in Österreich 19. und 20. Jahrhundert, Wien 2006, S. 49. 
Unternehmen erstmals Werbeabteilungen ein, das Berufsbild des Reklamefachmanns war im Entstehen. Praxisbezogene Handbücher und Einzelhandelszeitschriften setzten sich eingehend mit verkaufsfördernden Techniken auseinander. ${ }^{43}$

Zudem hatten sich die Einkommensverhältnisse verbessert, breitere Bevölkerungsteile konnten sich nun - in unterschiedlicher Ausprägung - am Massenkonsum beteiligen. Bedürfnisse wurden geweckt und hatten sich verschoben. Ausdruck dieser Entwicklung waren die um 1900 neu entstandenen Kauf- und Warenhäuser, die sich sozialer Schranken enthoben, Wünsche weckten und in Arrangement und Ausstattung Erlebniswelten kreierten, die zum Verweilen und Konsumieren anregten. ${ }^{44}$

$\mathrm{Zu}$ grundlegenden ökonomischen, aber auch kommunikationstechnischen Veränderungen kam es infolge des Ersten Weltkriegs. Einer beginnenden Prosperität im Zuge der kriegswirtschaftlichen Aufrüstung um 1915 folgte 1917 ein rascher Verfall der Produktion. Es mangelte an Rohstoffen und Arbeitskräften. Nahrungsmittel-, Brennstoffund Bekleidungsknappheit griffen um sich, wobei hier das logistische Unvermögen der Behörden mit ausschlaggebend war. ${ }^{45}$ Trotz der ökonomischen Bedingungen, die massive Rückgänge im Bereich des Werbemittelaufkommens zur Folge hatten, gab der Erste Weltkrieg der Wirtschaftswerbung enorme, nachhaltig prägende Impulse. Die Heimatfront war zu mobilisieren, Freiwillige wurden angeworben, die Bevölkerung musste zur Zeichnung zahlreicher Kriegsanleihen motiviert werden. Unter dem Einsatz modernster Werbemethoden, zu denen nun auch der Film zählte, steuerte die Politik die Massen. Auf allen Ebenen wurde für die Kriegsmaschinerie geworben. Der Erste Weltkrieg ist somit auch als „Urheber eines modernen Verständnisses von Massenkommunikation“ $\mathrm{zu}$ verstehen. ${ }^{46}$

Der Durchbruch der modernen Wirtschaftswerbung war durch die Dauerkrise der Zwischenkriegszeit nicht aufzuhalten. Eine durchgehende ökonomische Stagnation und Schrumpfung prägte die Jahre 1918 bis 1938. Auf die Hyperinflation, die 1922 ihren Höhepunkt erreichte, folgte eine Phase der Stabilisierung. 1929 setzte eine schwere Depression ein, von der sich die österreichische Wirtschaft nicht mehr erholen sollte. Die Massenarbeitslosigkeit war Ende 1934 mit 38,5 Prozent der unselbstständig Erwerbstätigen auf ihrem Höchststand und führte zu einem Schwund der

\footnotetext{
43 Morawetz, „Kontakt“ in der „Österreichischen Reklame“, S. 12-13, 69.

$44 \mathrm{Zu}$ der Innovationskraft der Warenhäuser in Wien und den zeitgenössischen Diskursen, die sich um die neuen Konsumwelten entsponnen, siehe: Schwarz, Werner Michael: Karneval der Waren. Warenhaus und Überschreitung, in: Breuss, Susanne/Eder, Franz X. (Hg.): Konsumieren in Österreich 19. und 20. Jahrhundert, Wien 2006, S. 71-85.

45 Sandgruber, Ökonomie und Politik, S. 319-322. Eigner/Helige: Wirtschafts- und Sozialgeschichte, S. 125-127.

46 Gries, Produkte \& Politik, S. 58, 67.
} 
Kaufkraft. ${ }^{47}$ Dennoch belief sich in den Jahren 1924 bis 1937 die Konsumquote real auf 80,6 Prozent. Somit lag in der Zwischenkriegszeit im Vergleich zum Jahr 1913 ein etwas höherer Lebensstandard vor, obwohl das reale Inlandsprodukt im Durchschnitt niedriger lag als im letzten Vorkriegsjahr. ${ }^{48}$

Insgesamt war die Periode 1918 bis 1938 aus konsumhistorischer Perspektive von Widersprüchlichkeiten geprägt: Einerseits hatte die Mehrheit der Österreicherinnen und Österreicher mit Mangel, oft sogar Armut, zu kämpfen. Andererseits wurde per Massenmedien einem prononcierten Konsumhedonismus gefrönt und einer Vielfalt an Konsumversprechen Raum gegeben. ${ }^{49}$ Die Produktkommunikation erlebte in der Zwischenkriegszeit einen quantitativen und qualitativen Aufschwung. ${ }^{50}$ Die Reklame war allgegenwärtig und sprach breite Teile der Bevölkerung an. Produkte, die bislang finanzkräftigen Schichten vorbehalten waren (z. B. kosmetische Erzeugnisse), wurden nun potenziellen Käufern des Kleinbürgertums und der Arbeiterschaft angeboten. Die Produktkommunikation öffnete sich „nach unten“, eine „Demokratisierung der Produktwahrnehmung“, wie Rainer Gries in seinen Studien konstatierte, setzte ein. ${ }^{51}$ Die Dauerkrise ließ werbetreibende Unternehmen umdenken, neue Strategien entwerfen und öffnete den Blick für neue Methoden der Vermarktung. ${ }^{52}$ Eine Professionalisierung der Wirtschaftswerbung war die Folge.

47 Weber, Fritz: Hauptprobleme der wirtschaftlichen und sozialen Entwicklung Österreichs in der Zwischenkriegszeit, in: Kadrnoska, Franz (Hg.): Aufbruch und Untergang. Österreichische Kultur zwischen 1918 und 1938, Wien/München/Zürich 1981, S. 593. Sandgruber, Ökonomie und Politik, S. 369-370, 383-384.

48 Sandgruber, Ökonomie und Politik, S. 365.

49 Eder, Franz X.: „Man lebte damals von der Hand in den Mund“. Zur Konsumgeschichte Wiens von 1920 bis 1945, in: Domenig, Roland/Linhart, Sepp (Hg.): Wien und Tokyo, 1930-1945. Alltag, Kultur und Konsum, Wien 2007, S. 14.

50 Mit Beginn der 1920er-Jahre werden moderne Werbeformen und -strategien sowie deren psychologische Wirkungsweisen zunehmend in Fachkreisen diskutiert und fachspezifische Ausbildungen forciert. Gries, Rainer: Die Medialisierung der Produktkommunikation. Grundzüge eines kulturhistorischen Entwurfs, in: Knoch, Habbo/Morat, Daniel (Hg.): Kommunikation als Beobachtung, Medienwandel und Gesellschaftsbilder 1880-1960, München 2003, S. 117-119.

51 Vgl. Gries, Produkte \& Politik, S. 64-67.

52 Vgl. dazu: Berghoff, Hartmut: Marketing im 20. Jahrhundert. Absatzinstrument - Managementphilosophie - universelle Sozialtechnik, in: Berghoff, Hartmut (Hg.): Marketinggeschichte. Die Genese einer modernen Sozialtechnik, Frankfurt/New York 2007, S. 37. Gries, Medialisierung der Produktkommunikation, S. 117. 


\subsection{Institutionalisierung der österreichischen Werbebranche}

Der Verfall der öffentlichen Sittlichkeit schreitet unaufhörlich voran. Theater und Kino, Schaufenster, Reklame und Kolportage [...] ertöten den letzten Rest des natürlichen Anstandes und bahnen bolschewistischen Moralgrundsätzen den Weg. ${ }^{53}$

Dieser diffamierende Anwurf des Linzer Bischofs Johannes Gföllner, der sich mehrmals in ähnlicher Weise über die „schädliche Wirkung“ des modernen Kulturlebens äußerte und mitunter auch antisemitische Untergriffe nicht scheute, ${ }^{54}$ vermittelt eine in breiten Kreisen der Gesellschaft verankerte Ablehnung der werbenden Zunft. Unseriöse Arbeitspraktiken, marktschreierisches Gebaren und unlautere Werbebotschaften wurden den Reklametreibenden oftmals unterstellt. ${ }^{55}$ Umso mehr lag es im Interesse der österreichischen Werbeschaffenden, das öffentliche Image der Branche mittels organisierter Aufklärungsarbeit und klarer gemeinschaftlicher Darlegung der Aufgaben, Regeln und Ziele der Werbetreibenden nachhaltig positiv zu beeinflussen. Unter der Devise „Kampf um die Wahrheit und gegen die Pfuscherei“ suchte man durch eine Vielzahl an Maßnahmen (Vorträge, Pressearbeit, Einrichtung einer Reklameberatungsstelle etc.), das Vertrauen in das Reklamewesen zu stärken. ${ }^{56}$

Zur Entwicklung eines Standesbewusstseins und um ein starkes, geschlossenes Vorgehen - etwa gegen die staatlichen Besteuerungssysteme - zu sichern, organisierte sich die österreichische Werbebranche Mitte der 1920er-Jahre in Interessenverbänden. ${ }^{57}$ Ein erster überregionaler Versuch eines kooperativen Zusammenschlusses erfolgte bereits 1914. Mit der Gründung der „Freien Vereinigung für Reklame-Kunst und Wissenschaft" verbanden Reklametreibende des deutschsprachigen Raums den Wunsch, Werbeforschung zu betreiben, die Ausbildung qualifizierter Fachkräfte zu forcieren und die Werbekunst zunehemnd zu ästhetisieren. ${ }^{58} 1922$ konstituiert sich als Zweigstelle des Wiener Kaufmännischen Vereins die „Österreichische Gesellschaft

53 Österreichische Reklame, „Johannes Maria und die Reklame“, Nr. 8, Februar 1928, S. 32.

54 Vgl. Moser, Karin: „Eine unerhörte jüdische Frechheit“ - JEW sÜss, in: Moritz, Verena/Moser, Karin/Leidinger, Hannes: Kampfzone Kino. Film in Österreich 1918-1938, Wien 2008, S. 322.

55 Vgl. u. a.: Österreichische Reklame, „Wir arbeiten!“, Nr. 1, November 1926, S. 3.

56 Österreichische Reklame, „Der Aufstieg der Reklame vom Pfuschertum zur Wissenschaft“, Nr. 1, November 1926, S. 4. Kontakt, „Die letzte Entwicklung und die nächsten Aufgaben der Reklame“, Nr. 1, Jänner 1931, S. 3 f.

57 Vgl. dazu auch: Österreichische Reklame, „Reklameunterricht“, Nr. 12, April 1928, S. 7.

58 Unter den Mitgliedern der Vereinigung fanden sich u. a. auch Vertreter aus Amsterdam, Prag, Moskau und Pilsen. Vgl. dazu: Ruben, Paul (Hg.): Die Reklame - ihre Kunst und Wissenschaft, Band 2, Berlin 1914. Zit. nach: Pernsteiner, Silvia Maria: Zum Schutz gegen Exzesse, Ausbeutungen und Monopolstellungen: Die Anfänge des organisierten Reklamewesens in Österreich, Dipl., Wien 2008, S. 33-34. 
für Reklamewesen“, über die Verbandsstatuten und die Tätigkeit dieser Organisation liegen jedoch keinerlei weiteren Aufzeichnungen vor. ${ }^{59}$

1925 bis 1927 erreichte die Institutionalisierung der Branche mit der Schaffung einer Reihe an Interessenvertretungen ihren Höhepunkt. Ausgangspunkt war vor allem die hohe Besteuerung des Inseraten- und Plakatwesens, welche die Wiener Unternehmer dazu veranlasste, zur „energischen Selbsthilfe“ ${ }^{\mathrm{zu}}$ greifen. ${ }^{60}$ In der Folge wurde im Jänner 1925 der „Schutzverband der Reklametreibenden Österreichs“ (SVRTÖ) ${ }^{61}$ errichtet, der sich u. a. eine Verringerung der staatlichen Abgaben, eine Verbesserung der fachlichen Ausbildung sowie die Schaffung einer Reklameberatungsstelle zum Ziel setzte. ${ }^{62}$ Letztere sollte Informationen über den Wert der erbrachten Werbeleistung und die Angemessenheit der verlangten Preise gewährleisten. Nach wenigen Jahren konnte die Auskunfts- und Interventionsstelle bereits eine erfolgreiche Bilanz aufweisen. Eine Vielzahl an Streitfällen zwischen Verbandsmitgliedern und einzelnen Reklameunternehmungen bzw. Zeitschriftenverwaltungen konnte bereinigt und beigelegt werden. ${ }^{63}$

Hervorgehend aus der Ortsgruppe Wien des Verbandes Deutscher Reklamefachleute, etablierte sich mit der Gründung des „Verbandes Österreichischer Reklamefachleute“ (VÖR) im Juli 1925 eine weitere bedeutende Standesorganisation der Branche. Als Ziele definierte der VÖR, sich für die Anerkennung der Leistungen der Zunft in der Öffentlichkeit einzusetzen, das Verständnis für die Arbeit der Werbetreibenden aktiv zu fördern, Standes- und Berufsinteressen zu wahren sowie den Ausbau und die qualitative Hebung der Aus- und Weiterbildungsmöglichkeiten voranzutreiben. ${ }^{64}$ Mit den Verbandsorganen Kontakt und Österreichische Reklame verfügten der SVRTÖ und der VÖR über Fachzeitschriften, die regelmäßig über Bestrebungen und Erfolge der beiden Institutionen wie auch über fachspezifische Veranstaltungen und neue Perspektiven der Werbewirtschaft, Werbekunst und Werbewissenschaft berichteten. Rege Verbindungen pflegten die Vereinigungen zudem mit branchennahen Organisationen, wie etwa dem 1925 begründeten „Österreichischen Verband der Markenartikelindustrie“ oder dem sich 1927 konstituierenden „Bund Österreichischer

59 Siehe dazu die Forschungsergebnisse von Pernsteiner, Schutz gegen Exzesse, S. 37-39.

60 Morawetz, „Kontakt“ in der „Österreichischen Reklame“, S. 36.

611928 erfolgte die Umbenennung der Vereinigung in „Österreichischer Reklameschutzverband“. Siehe: Kontakt, „Unsere Generalversammlung“, Nr. 12, April 1928, S. 7.

62 Ebd., 42-43. Siehe auch: Der Handel, „Schutzverband der Reklametreibenden Österreichs“, Nr. 10, Dezember 1924, S. 2. Der Handel, „Wie kann die Reklame verbessert und verbilligt werden?“, Nr. 20, Februar 1925, S. 6.

63 Kontakt, „Bevorstehende Errichtung einer Reklameberatungsstelle“, Nr. 16, September 1928, S. 6; Kontakt, „Ein Jahr im Dienste der reklametreibenden Geschäftswelt“, Nr. 12, S. 1-2.

64 Österreichische Reklame, „Wir arbeiten!“, Nr. 1, November 1926, S. 3. Pernsteiner, Schutz gegen Exzesse, S. 76. 
Gebrauchsgraphiker“. ${ }^{65}$ Das in den Jahren 1927 und 1928 eingerichtete Reklamewissenschaftliche Institut ${ }^{66}$ verwies ebenso wie die Berichterstattung der zeitgenössischen Branchenmagazine auf eine vermehrte methodische Auseinandersetzung mit den verschiedenartigsten Aspekten der Produktkommunikation.

\subsection{Verwissenschaftlichung und Professionalisierung der Produktkommunikation}

Bereits gegen Ende des 19. Jahrhunderts erschien eine Fülle an Publikationen und fachspezifischen Beiträgen, die sich dem Reklamewesen widmeten. Vor allem praktische Ratgeber, die direkte Konsumentenwerbung und die Gestaltung des Verkaufsorts ins Zentrum stellten, dominierten den Markt. ${ }^{67}$ Anfang des 20. Jahrhunderts erreichten neue, methodisch durchdachte Ansätze der Werbemittelgestaltung und -streuung den deutschsprachigen Raum. Forscher aus dem Bereich der Betriebslehre setzten sich erstmals mit Werbewirkungsmechanismen auseinander, unter ihnen der österreichische Nationalökonom, Jurist und Politiker Viktor Mataja. ${ }^{68}$ Sein 1910 veröffentlichtes Werk Die Reklame. Eine Untersuchung über Ankündigungswesen und Werbetätigkeit im Geschäftsleben galt als Standardwerk der historischen deutschsprachigen Werbewissenschaft und machte ihn zu einem der Pioniere der Verwissenschaftlichung und Professionalisierung der Reklame. ${ }^{69}$

Einen reellen Auftrieb erlebten die Werbewissenschaften in den 1920er-Jahren. Die Hyperinflation der Nachkriegszeit leitete eine Neuorientierung hinsichtlich der Bewertung von Kosten, Organisation und Nutzen ein. Betriebliche Rationalisierungsmaßnahmen, die eine Optimierung der Arbeitsabläufe und eine Senkung der

65 Heimisches Schaffen, „Zehnjahrfeier des Verbandes der Markenartikelindustrie“, Nr. 1, 2.7.1935, S. 7. Morawetz, „Kontakt“ in der „Österreichischen Reklame“, S. 15, 37, 46.

66 Österreichische Reklame, Nr. 14, Juli 1928, S. $12 f$.

67 Vgl. dazu auch: Spiekermann, Uwe: „Der Konsument muß erobert werden!“. Agrar- und Handelsmarketing in Deutschland während der 1920er und 1930er Jahre, in: Berghoff, Hartmut (Hg.): Marketinggeschichte. Die Genese einer modernen Sozialtechnik, Frankfurt/New York 2007, S. 143.

68 Mataja war in den Jahren 1908, 1909 und 1911 Handelsminister, 1918 bekleidete er das Amt des Ministers für soziale Fürsorge. Zudem war er als Universitätsprofessor in Wien und Innsbruck tätig. 1914-1917 und 1919-1922 fungierte er als Präsident der Statistischen Zentralkommission. Mataja war des Weiteres Mitglied des Ehren-Präsidiums der „Freien Vereinigung für Reklame-Kunst und Wissenschaft“ sowie Präsident der „Österreichischen Gesellschaft für Reklamewesen“. Vgl. auch Morawetz, „Kontakt“ in der „Österreichischen Reklame“, S. 13. Pernsteiner, Schutz gegen Exzesse, S. 33, 37-38, 151.

69 Silberer, Günter/Mau, Gunnar: Anfänge und Geschichte der Werbewirkungsforschung, in: Berghoff, Hartmut (Hg.): Marketinggeschichte. Die Genese einer modernen Sozialtechnik, Frankfurt/ New York 2007, S. 232.

Pernsteiner, Schutz gegen Exzesse, S. 151. Österreichische Reklame, Nr. 1, November 1926, S. 4. Morawetz, „Kontakt“ in der „Österreichischen Reklame“, S. 13. 
Produktionskosten forcierten, stießen auch in Österreich auf verstärktes Interesse, was etwa auch in der Gründung des „Österreichischen Kuratoriums für Wirtschaftlichkeit“ im Jahr 1928 seinen Ausdruck fand..$^{70}$ Die Krise als Chance zu erkennen, um aktiv zu werden, Umstrukturierungen vorzunehmen und neue Techniken zu erproben, war Tenor einer Debatte, die auch die Reklamebranche erfasste. Wirtschaftliches und zielgerichtetes Werben unter der Anwendung von Kontrollmechanismen wurde als neue Leitlinie ausgegeben. ${ }^{71}$ In diesem Sinne wurde 1928 innerhalb des SvRTö eine „Efficiency-Sektion“ eingerichtet. Den Begriff „Efficiency“ definierte man als „das Bestreben, innerhalb der jeweils herrschenden Arbeitsverhältnisse und Verdienstmöglichkeiten, durch Anwendung geeigneter Maßnahmen und Methoden [...] den größtmöglichen, effektiven Verdienst für die Leistung zu erzielen“. Anders als bei Fragen der Rationalisierung und Organisation, stünden beim „EfficiencyGedanken“ weniger „technische Aufgaben“ bzw. „Betriebs- und Verkaufslehre“, sondern vielmehr „psychologische Momente“ und besonders der Mensch im Mittelpunkt. Die Reklame wäre fortan in „Theorie und Praxis wissenschaftlich auszurichten“, wobei Werbepsychologie und Marktanalyse Anwendung finden müssten, um den Erfolg zu sichern. ${ }^{72}$

Anleihe nahm die Efficiency-Bewegung bei US-amerikanischen Forschungen, die in den deutschsprachigen Raum Eingang fanden und mitunter modifiziert wurden. Schon nach 1890 entstanden in den Vereinigten Staaten theoretisch fundierte Werbe- und Marketingstudien. Die Größe und Komplexität des Marktes verstärkte den Bedarf an wissenschaftlich gestützten Methoden der werbe- und marketingtechnischen Durchdringung. Zwischen den USA und Europa erfolgte ein reger Austausch in Hinblick auf neue Ansätze im Bereich der Werbepsychologie sowie der Markt- und Motivforschung. Ab den 1930er-Jahren beeinflussten europäische Soziologen, Psychologen, Nationalökonomen und Architekten nachhaltig die USamerikanische Konsumforschung, unter ihnen etwa auch die Österreicher Paul Lazarsfeld, Victor Gruen oder Ernest Dichter. ${ }^{73}$

70 Sandgruber, Ökonomie und Politik, S. 363. Spiekermann, „Der Konsument muß erobert werden!“, S. 138.

71 Österreichische Reklame, „Wirtschaftlicher werben! Wie man planlosem Werben ein Ende macht.“, Nr. 14, Juli 1028, S. 19. Verkaufspraxis, „Was tun? - Was tun!“, Nr. 2, November 1932, S. 102-105.

72 Kontakt, „Die Efficiency-Bewegung in Österreich“, Nr. 10, Februar 1928, S. 11-13. Kontakt, „Efficiency - Der Weg zum Erfolg“, Nr. 17, Oktober 1928, S. 3-6.

73 Berghoff, Marketing im 20. Jahrhundert, S. 31-37. Einen intensiven wissenschaftlich geprägten Informationstransfer belegen auch zahlreiche Artikel in den österreichischen Fachzeitungen. Siehe z. B.: Österreichische Reklame, „Marktanalyse“, Nr. 10, April 1928, S. 15. Österreichische Reklame, „Von der Wortwahrnehmung zum Kaufentschluss. Eine psychologische Skizze“, Nr. 3, 1929, S. 7-9. Kontakt, „Rationalisierung von Verkauf und Reklame“, Nr. 5/6, Mai/Juni 1929, S. 1-4. Kontakt, „Marktanalyse als Werkzeug der Reklame“, Nr. 10, Oktober 1930, S. 36-38. Contact, „Wirtschaftswerbung. Öffentliche Werbung“, Nr. 4, April 1936, S. 8-10. 
Das Aufkommen eines derart geschulten Experten-Pools in Österreich war auch auf die Intensivierung der Forschungs- und Ausbildungsaktivitäten in den werbewissenschaftlichen Disziplinen zurückzuführen. Vor allem erarbeiteten das „Psychotechnische Institut der Industriellen Bezirkskommission in Wien“, das „Reklamewissenschaftliche Institut“ an der Hochschule für Welthandel, das „Psychologische Institut“ der Universität Wien sowie die „Österreichische Werbepsychologische Forschungsstelle“ (ÖWPF) Werbewirkungsstudien. ${ }^{74}$ Die beiden letztgenannten Institutionen zeichneten sich im Speziellen durch innovative Vorgehensweisen und Arbeiten aus. Am von Karl Bühler in enger Zusammenarbeit mit seiner Frau Charlotte geleiteten Psychologischen Institut der Universität Wien wurden erstmals in Österreich Untersuchungen und Versuchsreihen zum Thema Film durchgeführt, wobei man hier auch didaktische Fragestellungen einbezog. ${ }^{75}$ Eine Reihe von Dissertationen, die das Medium Film auf wahrnehmungs-psychologischer und kommunikationstheoretischer Ebene analysierten, belegen die fortschrittliche Forschungstätigkeit in diesem neuen Feld. ${ }^{76}$

Die Assistenztätigkeit des Soziologen Paul Lazarsfeld am Psychologischen Institut war Ausgangspunkt für die Errichtung einer der bedeutendsten Forschungseinrichtungen der Zwischenkriegszeit. Mit Unterstützung von Karl und Charlotte Bühler begründete Lazarsfeld 1931 mit der ÖWPF ein kleines, fortschrittlich agierendes Marktforschungsinstitut, wobei das Kuratorium der Einrichtung aus politischer und wirtschaftlicher Sicht hochkarätig besetzt war. ${ }^{77}$ Die Mitarbeiterinnen und Mitarbeiter der sich als „Stelle für Motivforschung“ bezeichnenden Institution führten in den Jahren

74 Morawetz, „Kontakt“ in der „Österreichischen Reklame“, S. 98.

75 Der Wiener Film, Nr. 32, 15. Dezember 1936. Neue Freie Presse, „Filmnachrichten“, 20.5.1936, S. 8 .

76 Vgl. etwa: Spiel, Hilde: Versuch einer Darstellungstheorie des Films, Dissertation, Wien 1935. Ohmes, Richard: Die Bedeutung des Kinos für Kinder und Jugendliche, Dissertation, Wien 1935. Lassner, Rudolf: Theater- und Kinobesuche. Eine psychologische Analyse, Dissertation, Wien 1936. Winter, Leopold: Komik im Film, Dissertation, Wien 1938.

77 Im Präsidium fanden sich neben Karl Bühler, der Präsident der Wiener Handelskammer und somit der ehemalige Bundeskanzler Ernst Streeruwitz, der Generalsekretär der Wiener Kammer für Arbeiter und Angestellte und der Generalsekretär der Niederösterreichischen Landwirtschaftskammer. Im Beirat vertreten waren u. a. Günther Bandat, Geschäftsführer des Kuratoriums für Wirtschaftlichkeit, Rudolf Brichta, Sekretär des Gremiums der Wiener Kaufmannschaft, Richard Butschowitz, Chefadministrator, Präsident des Verbandes der Reklamefachleute, F.H. Davis, Vizepräsident des Reklameschutzverbandes sowie die Direktoren der Firmen Mautner-Markhof, Meinl, Delka, Bally, Gerngroß sowie der Städtischen Versicherung. Vgl. dazu: Neurath, Paul: Paul L. Lazarsfeld in Emigration und (teilweiser) Remigration, in: Stadler, Friedrich (Hg.): Vertriebene Vernunft II. Emigration und Exil österreichischer Wissenschaft. Internationales Symposium 19. bis 23. Oktober 1987 in Wien, Wien/München 1988, S. 361-362. Maria Jahoda, 1907-2001. Pionierin der Sozialforschung. Katalog zur Ausstellung des Archivs für die Geschichte der Soziologie in Österreich an der Universitätsbibliothek Graz vom 3. Juni bis 2. August 2002, Graz 2002, S. 41. 
1931 bis 1935 zahlreiche auf psychologischen und sozialpsychologischen Forschungsmethoden basierende Untersuchungen über den alltäglichen Verbrauch und das damit korrelierende Käuferverhalten durch. Anhand standardisierter Fragebögen wurden Ereignisverläufe erlebter Kaufhandlungen verfolgt und eine Reihe an „Kaufbiographien“ dokumentiert. ${ }^{78} \mathrm{Zu}$ den untersuchten Produkten zählten etwa Tee, Kaffee, Bier, Zucker, Essig, Schokolade, Blumen, Schuhe, Hemden, Parfüms sowie eine Vielzahl kleiner Haushaltsgüter. Als Auftraggeber fungierten große österreichische und internationale Unternehmen, wie beispielsweise Ankerbrot, Julius Meinl, Delka, Herzmansky, Hardtmuth, Titze oder das Budapester Fremdenverkehrsbüro. Studien über den Teekonsum in Wien, über die Chancen von Fertigkaffee-Erzeugnissen in der österreichischen Bundeshauptstadt, über den Berliner Futtermarkt und Milchkonsum sowie über den Handschuhabsatz in den Zürcher Warenhäusern wurden ebenso erstellt wie Vergleichsprofile des proletarischen und des Mittelklasse-Verbrauchers. Zudem beauftragten renommierte internationale wissenschaftliche Organisationen, wie etwa das Frankfurter Institut für Sozialforschung, die ÖWPF mit der Erfassung von Daten. ${ }^{79}$

Die bekanntesten Arbeiten der ÖwPF sind zweifellos die Hörerbefragungen für den österreichischen Rundfunk 1931 sowie die nicht kommerzielle Studie über die Auswirkungen der Langzeit-Arbeitslosigkeit im Marienthal 1933. ${ }^{80}$ Weniger beachtet wurde bislang eine Untersuchung bezüglich der Wirkung von Reklamemitteln, die 1934 vorlag und auf Besucher-Befragungen anlässlich der Wiener Frühjahrsmesse 1933 basierte. Die Rechercheure der ÖWPF hielten u. a. Faktoren wie Alter, Geschlecht, Beruf, sozialökonomische Stellung, Persönlichkeitstyp und Kaufgewohnheiten fest. In Hinblick auf die Wirksamkeit der Werbemittel konnte der effektive Grad nach Anzahl der Nennung in absteigender Reihung ermittelt werden: Plakate (67,6 Prozent), Lichtreklame (41,1 Prozent), Straßenbahnreklame (31,4 Prozent), Zeitungsreklame (29,4 Prozent), Kinoreklame (21,9 Prozent), Wurfsendungen (11,9 Prozent). ${ }^{81}$ Trotz erfolgreicher und

78 Busch, Emil Walter: Faktor Mensch. Formen angewandter Sozialforschung der Wirtschaft in Europa und den USA, 1890-1950, Konstanz 2006, S. 391, 396. Neurath, Lazarsfeld in Emigration, S. 362. Maria Jahoda, 1907-2001, S. 39-41.

79 Pollak, Michael: Paul F. Lazarsfeld - Gründer eines multinationalen Wissenschaftskonzerns, in: Langenbucher, Wolfgang R. (Hg.): Paul Felix Lazarsfeld - Leben und Werk. Anstatt einer Biografie, Wien 2008, S. 162. Busch, Faktor Mensch, S. 396. Maria Jahoda, 1907-2001, S. 68. Neurath, Paul: Paul Lazarsfeld und die Institutionalisierung der empirischen Sozialforschung: Ausfuhr und Wiedereinfuhr einer Wiener Institution, in: Langenbucher, Wolfgang R. (Hg.): Paul Felix Lazarsfeld Leben und Werk. Anstatt einer Biografie, Wien 2008, S. 201-202.

80 Vgl. dazu Pollak, Paul F. Lazarsfeld, S. 162. sowie: http://agso.uni-graz.at/marienthal/, 28.10.2012.

81 Contact, „Reklame und Käufer“, Nr. 4, April 1934, S. 19-23. 
international anerkannter Studien wurde die ÖWPF angesichts politischer Aktivitäten, die sich gegen das austrofaschistische System richteten, im März 1937 aufgelöst. ${ }^{82}$

Parallel zur Etablierung der Reklamewissenschaften in Österreich stieg das Engagement, auch die fachkundige Aus- und Weiterbildung voranzutreiben bzw. diese nach modernsten Standards auszurichten und eine „spezifisch österreichische Lehrmethode“ zu entwickeln. ${ }^{83}$ Ein erster Versuch, das Unterrichtsfach „Reklame“ in den Lehrplan zu integrieren, erfolgte bereits in den Jahren 1908 bis 1910 an der privat geführten Handelsschule Allina. ${ }^{84}$ Es sollte für lange Zeit die einzige Unternehmung dieser Art in Österreich sein. Erst im Verlauf der 1920er-Jahre gab es verschiedenartige Anstrengungen, reklametechnische Schulungen durchzuführen. 1926 etwa wurden seitens der Abteilung für Nach- und Umschulung der „Industriellen Bezirkskommission in Wien" Reklame- und Propagandakurse zur Weiterbildung arbeitsloser Angestellter der Industrie und des Handels abgehalten. 1927 warb der Österreichische Reklameschutzverband für eine Schulung unter dem Titel „Reklame-Psychologie in Theorie und Praxis“. ${ }^{85}$ Im selben Jahr wurden, beruhend auf einer Initiative der „Reklamewissenschaftlichen Gesellschaft“ unter Präsident Viktor Mataja und dem VÖR, sogenannte „Reklamehochschulkurse“ begründet, die sich als abendliches Fortbildungsangebot an Fachkräfte der Branche wandten. Dieser Lehrgang wurde Ende der 1920er-Jahre vom „Staatlichen Reklameausbildungskurs“ abgelöst, der fortan von der Hochschule für Welthandel durchgeführt werden sollte, sich vermehrt der Praxis widmete und in sinnreicher Form auf den Erwerb einzelner Kenntnisse aufbaute. ${ }^{86} 1931$ zählte das Fach „Reklame“, in den unterschiedlichsten Spezialausrichtungen, zum Unterrichtsgegenstand an den branchennahen Schulen und Universitäten. ${ }^{87}$

82 Bereits 1935 wurde die ÖWPF aufgelöst, stattdessen wurde die von Maria Jahoda geleitete „Arbeitsgemeinschaft der Österreichischen Wirtschaftspsychologischen Forschungsstelle“ begründet. Die austrofaschistischen Machthaber verdächtigten die Vertreter der Institution sich politisch subversiv zu betätigen und verhafteten im Jahr 1937 zahlreiche ihrer Mitglieder und Sympathisanten, unter ihnen auch Karl Bühler. Maria Jahoda wurde schließlich wegen illegaler politischer Betätigung zu drei Monaten Haft und anschließende „Anhaltehaft“ verurteilt. Aufgrund internationaler Interventionen entließ man Jahoda noch im Jahr 1937 und verwies sie des Landes. Siehe dazu: Busch, Faktor Mensch, S. 399.

83 Siehe dazu die Ausführungen in: Kontakt, „Zur Frage der Reklameschulen“, Nr. 14/15, JuliAugust 1928, S. $4 \mathrm{f}$.

84 Morawetz, „Kontakt“ in der „Österreichischen Reklame“, S. 14, 78.

85 Ebd., S. 73.

86 Ebd., 74-77. Österreichische Reklame, „Reklameunterricht“, Nr. 14, Juli 1928, S. 11.

87 1936/37 wurde an der Hochschule für Welthandel beispielsweise ein Kurs zum Thema „Psychologie der Reklame“ abgehalten, im darauffolgenden Studienjahr wurden Seminare über Marktforschung und Reklamepsychologie angeboten. Vgl. Pernsteiner, Schutz gegen Exzesse, S. 138 f. Kontakt, „Die letzte Entwicklung und die nächsten Aufgaben der Reklame“, Nr. 1, Jänner 1931, S. 3-9. 
Ein zentrales Thema der Bildungspläne war stets der zielgerichtete Einsatz und die funktionell-ästhetische Gestaltung der Werbemittel. Obwohl bereits 1926 im Zuge der Debatte hinsichtlich einer Reformierung des kaufmännischen Unterrichtswesens auch die „Filmreklame“ als Teil der Außenwerbung angeführt worden war, ${ }^{88}$ fehlte sie in den von der Branche skizzierten Ausbildungsprogrammen gänzlich. ${ }^{89}$ Dies entsprach auch der Wahrnehmung des „werbenden Films“ in den Branchenblättern. Es dominierten Berichte über die Printreklame, die Schaufenstergestaltung und den Einsatz von Leuchtelementen. ${ }^{90}$ Eine vermehrte Auseinandersetzung mit dem Medium Film erfolgt erst im Verlauf der 1930er-Jahre. Trotzdem lassen sich Frühformen des Werbefilms schon zu Beginn der Kinematographie im Vorführungszyklus der Varietéund Wanderkinobetriebe ausmachen. Sie bereiteten dem neuen Reklamemittel den Weg.

88 Kontakt, „Die Neuordnung des kaufmännischen Bildungswesens“, Nr. 2, März 1926, S. 6-7.

89 Vgl. - etwa: Österreichische Reklame, „Reklamewissenschaftliches Institut“, Nr. 14, Juli 1928, S. 12 f. Werbe-Winke, „Auch Reklame will gelernt sein!“, Nr. 5, 1. Mai 1935, S. 20 f.

90 Vgl. hierzu auch die Analyseergebnisse von Morawetz, „Kontakt“ in der „Österreichischen Reklame“, S. 109, 114. 